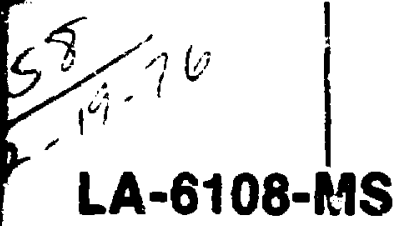

Informal Report

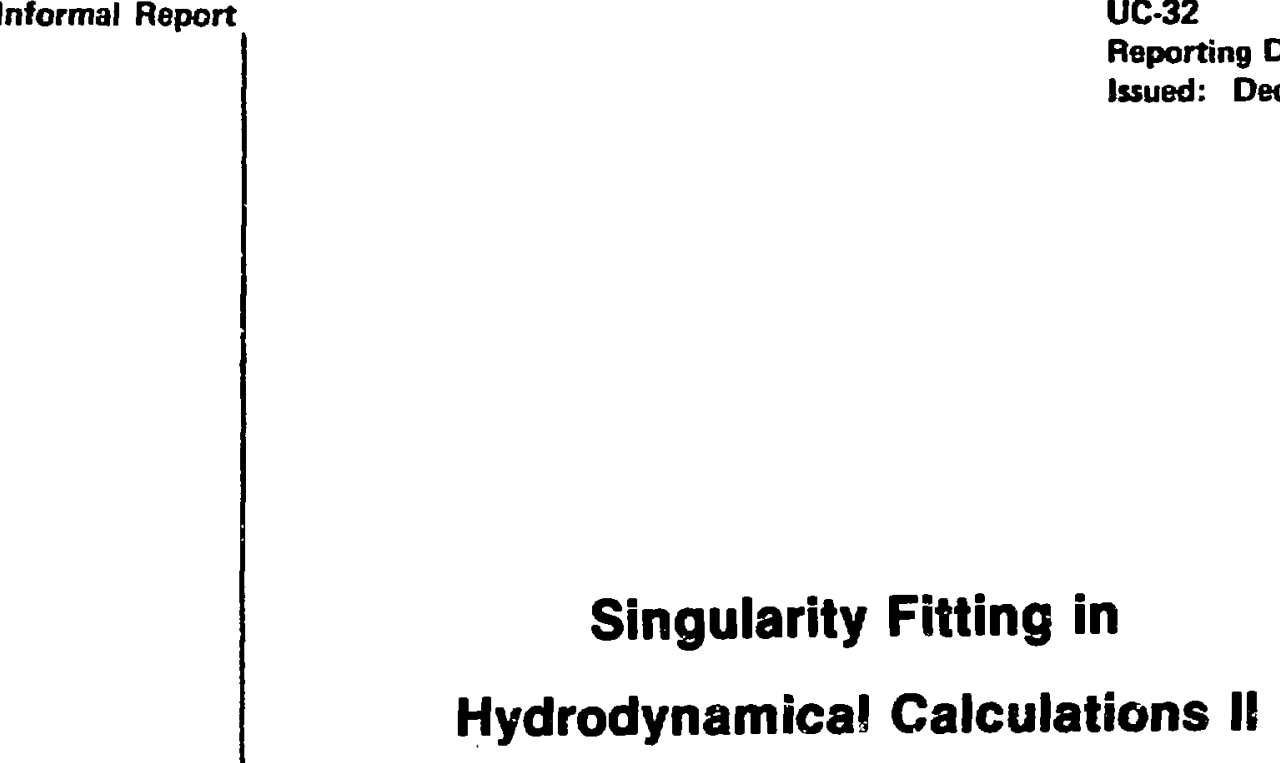

by

R. D. Richtmyer*

R. B. Lazarus

Norece 7 acount of work sponsored by the United States Gover roment. Nenther the United States nos the Unirod Sutes Encray Research and Serdopgent Adnimastretion, nas any of their emphyees, not sny of thes contrartors sutisontracturs. of thes es wemnty. expien ar ingled.

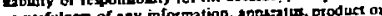
or verfuines of any trets infringe frivtely owned rights.

of the Universify of California

LOS ALAMOS, NEW MEXICO 87545

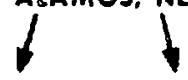

An Affirmative Action/Equal Opportunity Employer 
In the interest of prompt distribution, this report was not edited by the Technicel Information staff.

Printed in the United Statea of America. Available fram National Technical Inform mition Service

U.S. Department of Commerce

5285 Port Royal Rond

Springueld, VA 22151

Price: Printed Copy $\mathbf{4 . 5 0}$ Microfiche $\mathbf{8 2 . 2 5}$

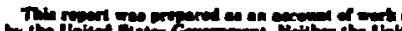

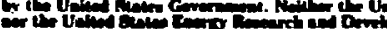

and

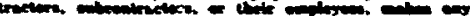

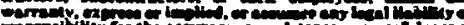

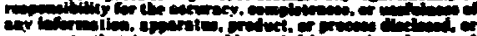

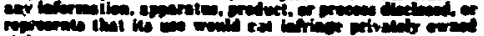

inte. 


\section{CONTENTS}

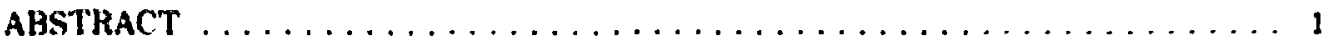

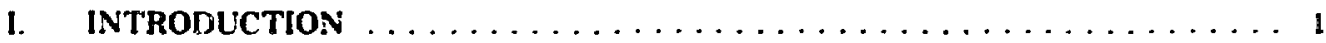

11. THE RAREFACTION (CAVITY COLLAPSE) RROBLEM .......... 2

1II. DIFFERENCE EQUATIONS FOR THE SMUOTH PART OF THE FLOW . . . 3

IV. THE FRES.SURFACE CONDITION $\ldots \ldots \ldots \ldots \ldots \ldots \ldots \ldots$

v. FITTING THE HEAD OF THE WAVE AND THE FAEE SURFACE . . . . . . 5

VI. SOME NUMERICAL RESULTS FOR THE RAREFACTION

PHASE PRIOR TO COLLAPSE $\ldots \ldots \ldots \ldots \ldots \ldots \ldots \ldots \ldots \ldots \ldots \ldots \ldots \ldots$

VII. SOME SELF.SIMILAR SOLUTIONS OF THE

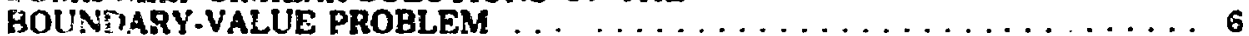

VIII. THE SIMILARITY SOLUTION AFTEH COLLPASE; THE

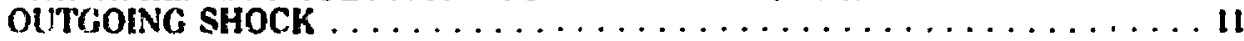

IX. THE FLUID-DYNAMICAL. EQUATIONS IN SIMILARITY VARIABLES . . . . 12

x. THF ASYMPTOTTC BEHAVIOR OF THE CAVITY.COLLAPSE PFOBLEM . . I4

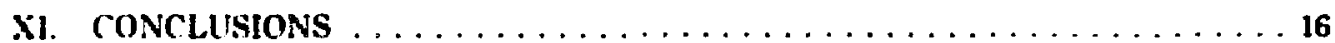




\title{
SINGULARITY FITTING IN \\ HYDRODYNAMICAL CALCULATIONS II
}

by

\author{
K.D. Richtmyer and R.B. Lazurus
}

\begin{abstract}
This is the second report in a series on the development of techniques for the proper handlink of singularittes in fluiddynumical calculations; the firct was called Itogress Report on the shosf-Fitling frojoct. This repert conteins six main results: (1) derivation of a free-surface condition, which reletes the acceleration of the surfuce with the rradient of the squste of the sound speed just behind it; (2) an accurate method for the early and middle stinges of the development of a rarefaction wave. two orders of maxnitude more eccurate than a simple direct method used for comparison; (3) the similerity theory of the collapsing free surface. where it is show'n that there is two-parameter family of selfsimilar solutions for $;=3.9$; (4) ihe similarity theory for the outgoink shock. which takes into account the entropy increase; (5) "zoominx" method for the study of the asymptotic lehavior of solutions of the full initial boundary-valuse problem: (6) comparison of two methods for determining the similaritg: parameter $\delta$ by $300 \mathrm{~m}$ inx. which shows that the second method is yreferred.

Future reports in the series will costain discussions of the selfsimilar solutions for this problem, end for that of the collapsing shoxk, in more detail and for the full range $(1, \infty)$ of $\gamma:$ the values of certain integrals related to neutronic and thermonuclear rates near callupse; and methods for fittink shocks. contact discontinuities. interfaces. and tree surfuces in two-dimensional Hows.
\end{abstract}

\section{INTRODUCTION}

Shuck-fitting metherds were developed in Los Alamos in 1948 for one-dimensional problems with spherical symmetry. for the special case in which thefe is just one primary shuck. whose position and veluxity are known at $t=0$, and which runs into presinusly undisturbed material. In spite of the simplifications, the method was sufficiently difficult for the enrly computers that, when ihe Hippo project was being plauned. in 1948. the pseudo-viscosity method was invented to replace shock fitting. ' When used with a great deal of care and - certain amount of good luck. the viscosity method can give gond results, but is quite risky at best ${ }^{2}$ and is seriously lacking in spatial resolution in multidimensional prublems. It has piven quite incorrrect results in a few cases. A sinall project was started here in 1974 to develup shock fitting further in one dimension and to extend it to two dimensions: Ref. 3 is a preliminary report on that work.

In the cousse of the shock-fitting studies, it hecame apparent that there are other singularities of nows which also ought to be treated by special methods, which will be cailed generally fitting methods. They include inierfaces, contact discon. tinuities, free surfaces, rarefaction heads and tails. shock interactions. corners. centers of symmetric collapse. and the like. That has led us to the follow. ing working principle as a basis for study: The finite 
difference methods ought to be used only for the smooth parts of the now. where the differential equations are strictly valid, and all other parts ought (1) be specially treated by whatever mixture of andiytic and numerical methods can be devised.

One advantage of that principle is that it gives one a lot of freedom in the choice of the finite difference method to be used in each of the smooth parts into which the flow is divided by the singularities. (The freedum is made use of in the particular problem to which this report is devoted by the cheice of special dependent variables suited to the development of a rarefaction wave.) In particular, the degree of dissinativity of the difference equations can be chosen Io satisfy Kreiss's theorem ${ }^{4}$ rather than with any idea of smearing out shocks.

Another advantage of that principle is that it removes the most serious disadvantage of the Fulerian formalism, namely the loss of precise loca:iun for material interfaces and other discrete surfaces.

It is recalled that one purpose of the Lax. Wendroff method was to fill a gap in the discussion of the viscosity method in Ret. 1. That discussion showed that the correct description of a flow with shocks is ublisinable. at least in principle. by first letting the spacing $\lambda \mathrm{x}$ of the computation net tend to zero and then letting the viscosity coefficient, or equivalentl'; the shock thickness d. tend to zero subsequently. That sidesteps the question of what happens if the limits are taken simultaneously, so that $J x$ and $d$ remain of the same order of magnitude, as they alway: are in practical calculations. The same question $i$; unanswered in nearly all the modern versions of the viscosity method.

The Lax-Wendroff method fills that gap in the discussion by conserving mass, momentum, and energy exactly, in a certain sense ${ }^{4}$ already for finite $\Delta x$. not merely in the limit as $\mathbf{A x}-\mathbf{0}$. Since the Rankine-Hugoniot jump conditions for a shock ane based on the conservation law:s, they also hold, in a sense, for finite $\mathbf{\Delta x}$.

Another purpose of the Lax. Wendroff method (posisibly seen most clearly in retrospect) was to clear up the confusion that existed concerning the dissipativity of a difference scheme and the dissipative terms in such a scheme. Difference schemes are usually anaiyzed, following von Ne: mann, by first linearizing, then treating the coefficients as con stants (a: legst in srall neighborhoods), anc then expanding the solution in a Fourier series or pourier integral. The time dependence of the Frurier coefficients is then determined by the difference equations. If the absolute value of a very Fourier cotifficient remains constant in time, as is the case for the differential equations when similariy treated. the scheme is called nondissipative. For any regsonable difference scheme. that mist he approximately true for the long and medium wavelength cumponents, but the short wavelength ones are often significantly damped, as $t$ increases. in which case the scheme is called dissipative.

It was formerly felt that difference methods for fluid dynamics ought to be nondissipative, because the differential equations are. However, it can be shown that any finite oufference scheme necess drily falsifies the phases of the short-wave components; hence. it is pointless to maintain their amplitudes. from the viewpoint of accuracy. That it is also pointless to maintain their amplitudes from the viewpoint of the conservation laws is shown by the Lax-Wendroff equations, which conserve mass, momentum. and energy exactly, but are dissipative. Lastly. Kreiss's theorem shows that a suitable ciegree of dissipativity. corresponding to a given degree of accuracy. guarantees stability against variability of the coefficients, when the von Neumann condition is satisfied.

When, as in the present work, difference equations are used only for the smooth part of the flow, the two main functions of the Lax. Wendroff method, conservation and dissipation, can be separated. We are interested only in the lat ter, hence need not require the equations to be in conservation-law form, but can apply the two-step Lax-Wendroff method of differencing directly to equations of the form

$$
\frac{\partial U}{\partial t}+A \frac{\partial U}{\partial x}=0
$$

for it is known that such differencing gives the amount of dissipation required by Kreiss's theorem.

\section{THE RAREFACTION (CAVITY COLLAPSE) PROBLEM}

At time $t=0$, a $\gamma$-law fluid is at rest under constant pressure in the region of space outside an empty spherical cavity. i.e., in the region $R>R_{0}$, where $B_{i}$ is the Eulerian radial coordinate. See Fig. 2-1. (The computer code was originally planned to handle also the corresponding plane and cylindrical problems, hut so far only the spherical one has been studied.) For $0<t<t_{11}$, where $t_{1}$ is the instant of collapse of the cavity, there is a rarefaction wave between an inward moving free surface at $R=\xi(t)$ and an outward moving head at $R=\eta(t)$, where $0<\xi(t)<R_{t n}$ and where $\eta(t)=R_{n}+c_{0} t, c_{n}$ being the sound speed in the initial state. For $t \ll i_{n}$, the spherical shell $\xi(t)<$ $R<\eta(t)$ occupied by the wave is "ery thin, and the rarefaction is approximately a plane simple wave ${ }^{5}$ in which the sound speed $c$ and the material speed $u$ 

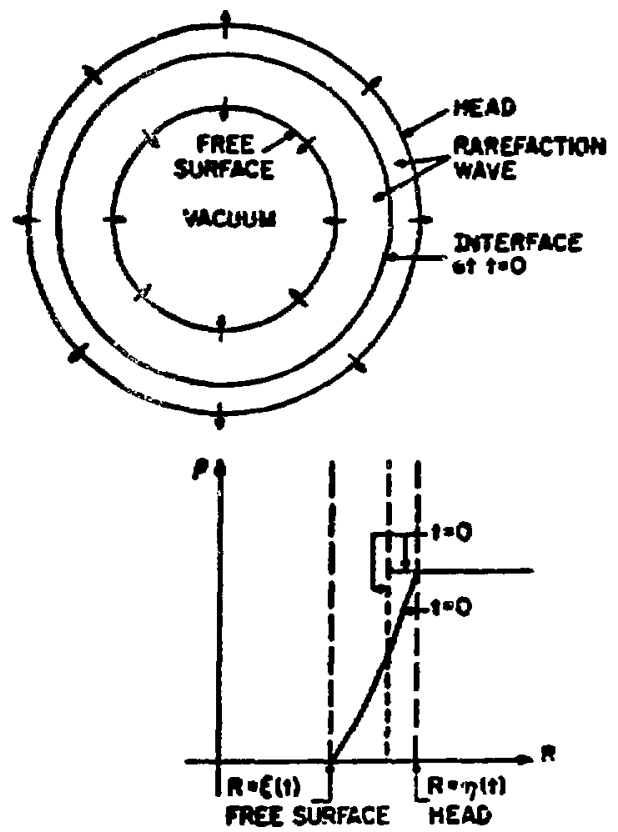

Fig. 2-1.

The cavity collapse program at, and shortly after. $t=0$.

vary linearly with $R$ across the shell. In that stage, the velocity of the free surface is given approximately by $\dot{\xi}(=\mathrm{d} \xi / d t)=-2 c_{\alpha} /(\gamma-1)$. At later times, the free surface accelerates inward until collapse occurs at $t=t_{n}\left|\xi\left(z_{n}\right)=0\right|$, at which time the pressure and density are instantaneously infinite at $R=0$. For $t>$ h. a shock front, whose position is denoted by $R=$ $r(t)$. proyresses out ivard from the center.

The flow has singularities as follows:

(1) $t \approx 0, R \approx R$. At $t=0$, the flow quantities are discontinuous across $R=R_{3}$. At early times thereafter. there is a thin centered rarefaction wave in an interval $|\xi, \eta|$ around $R_{0}$. Although the theory of such waves has: been well known in the plane approximation since the time of Riemann, the wave has to be regarded as a singularity frum the viewpoint of the finite difference equations, so long as its thickness is comparable with or less than the spacing $J R$ of the computational net.

(2) $t>0, R=\eta(t)=R_{n}+c_{n} t$. At the head of the rarefaction wave, the first derivatives of the flow quantities are discontinuous. A special fitting technique is used; it is described in Sec. V.
(3) $0<t<t_{0}, R=\xi(t)$. At the free surface, a special boundary condition is needed in addition to fitting. It is described in Sec. IV.

(4) $t \approx t_{\text {, }}$, but $t<t_{0}, R \approx 0$. The collapsing flow may be representable asymptotically by a selfsimilar flow of the kind described by Hunter ${ }^{6}-$ see also Ref. 7. This question is debated in Sec. X.

(5) $t \approx t_{0}$, but $t>t_{0}, R \approx 0$. The outgoing shock may also be representable asymptotically by a selfsimilar flow.

(6) $t>t, R=\zeta(t)$. Shock fitting, as described in Ref. 3, is required at the outgoing shock.

The main computationai problem, to which the latter part of this report is addressed. is how to turn the flow around, at $t \approx t_{t}$, and get the outgoing shock properly started.

\section{DIFFERENCE EQUATIONS FOR THE SMOOTH PART OF THE FLOW}

At early times, owing to the approximate plane simnle-wave character of the solution, it is natural to take the entropy $S$ per unit mass, the Reimann variable $\rho \mid=2 c /(\gamma-1)$ for a $q$-law gas . and the fluid velocity $u$. as the dependent variables, because those quantities vary linearly with $R$ in the simple wave, whereas the pressure $p$ and the density $p$ vary with a higher power of the distance from the free surface (see Nite following Eq. 3.8). The difterential equations for $S$. $\sigma$, and $u$ will next be derived from the Eulerian equations, which, for plane $(\alpha=1)$. cylindrical $(\alpha=2)$, and spherical $(\alpha=3)$ symmetry. are

$$
\begin{aligned}
& \left(\frac{\partial}{\partial \tau}+u \frac{\partial}{\partial R}\right)_{D}=-D \frac{1}{R^{a-1}} \frac{\partial}{\partial R}\left(R^{a-1} u\right), \mid \text { (a) } \\
& O\left(\frac{\partial}{\partial t}+u \frac{\partial}{\partial R}\right) u=-\frac{\partial P}{\partial P}, \\
& \left.D\left(\frac{\partial}{\partial t}+u \frac{\partial}{\partial R}\right) E=-p \frac{1}{R^{\alpha-1}} \frac{\partial}{\partial R}\left(R^{\alpha-1} v\right)\right)(c)
\end{aligned}
$$

$\xi$ is the internal energy per unit mass and is related to $p$ and $\rho$ by an equation of state. A consequence of Fq. 3.1 is the entropy condition

$$
\left(\frac{\partial}{\partial t}+u \frac{\partial}{\partial A}\right) s=0
$$


If $\widetilde{S}$ is any function of $\mathrm{S}$ (not depending on any other variables). then

$$
\left(\frac{\partial}{\partial t}+u \frac{\partial}{\partial R}\right) \xi=0 .
$$

We introduce as further thermodynamic quantity the Reimann variable

$$
\sigma=\left.\int \frac{d p}{\partial c}\right|_{S=\text { const }}=\sigma(\dot{S}, \rho) .
$$

For a y-law gas, we can take

$$
p=p\left(\tilde{\mathbf{S}}_{p}\right)=\tilde{\mathbf{S}}_{\boldsymbol{p}} \text { : }
$$

then

$$
\sigma=\sigma\left(\tilde{S}_{.}, \rho\right)=2 c /(\gamma-1)
$$

where

$$
c=c(\tilde{\mathbf{S}}, p)=\sqrt{\gamma \tilde{\mathbf{S}} \rho^{\gamma-1}} .
$$

The program is to take $\overline{\mathrm{S}}, \sigma$, and $u$ as dependent variables, define a vector

$$
U=(\mathbb{S}, \rho, u) \text {. }
$$

and desive the differential equations for $\widetilde{\mathcal{S}}, \rho, u$ in the form

$$
\mathrm{OU} / \partial t+\mathrm{ADU} / \mathrm{OR}=\mathrm{g},
$$

where $A$ is a $3 \times 3$ matrix. The first equation is already at hand: it is (3.2). A short calculation gives the system

$$
\begin{aligned}
& \left(\frac{\partial}{\partial t}+u \frac{\partial}{\partial R}\right) \xi=0 \\
& \left(\frac{\partial}{\partial t}+u \frac{\partial}{\partial R}\right) a+c \frac{\partial u}{\partial R}=-u c \frac{a-1}{R} \\
& \left.\left(\frac{\partial}{\partial t}+u \frac{\partial}{\partial R}\right) u+c \frac{\partial \sigma}{\partial R}+\left(\frac{1}{\partial} \frac{\partial p}{\partial S}-c \frac{\partial a}{\partial \xi}\right) \frac{\partial \xi}{\partial R}\right)_{(c)}^{(a)} \\
& =0 .
\end{aligned}
$$

In the cuefficient of $\partial \widetilde{S} / O R$ in Eq. (3.8c), the differentiations with respect to $\widehat{\mathrm{S}}$ are understood to be for fixed $\rho$.

Note: For a $\gamma$-law fluid, either of the variables $\sigma$ and $c$ can be eliminated by use of the equation $\sigma=$ $2 c /(\gamma-1)$. For a fluid with a non- $\gamma$-law equation of state, neither $a$ nor $c$ varies exactly linearly with distance across a plane sinple rarefaction wave, but a varies more nearly linearly than c, hence Eq. $(3.8)$ is the preferred form of the equation:" in whicli $c$ may be thought of as a function of $\sigma$ and $\widetilde{S}$.

As stated in the Introduction, we wish to use dissipative differeace equations of second-ordez accuracy. An easy way of abtaining them is the LaxWendroff two-step method of differencing (Ref. 4 , pp. 300-306). For equations of the form (3.7) we have, in the usual notation.

Step 1:

$$
\begin{aligned}
u_{j+1}^{n+1} & =\frac{1}{2}\left(u_{j+1}^{n}-u_{j}^{n}\right) \\
& -\frac{\Delta \tau}{2 \Delta s} \vec{A}^{n}\left(v_{j+1}^{n}-v_{j}^{n}\right)+\Delta \tau \vec{g}^{-n} .
\end{aligned}
$$

Step 2:

$$
u_{j}^{n+1}=u_{j}^{n}-\frac{\Delta t}{6 t} a^{n+1}\left(u_{j+1}^{n+1}-u_{j-j}^{n+1}\right)+\Delta t i^{n+1},
$$

where the overbars denute the appropriate spaicial averages of the matrix $A$ and the vector $g$. In the code, the following minor modification of these equations is used: In the system (3.8), $g$ appears only in the secend equation and can be included by rewriting the last term of the first member of that equation (for $\alpha=3$ ) as

$$
\frac{c}{R^{2}} \frac{\left(R^{2} u\right)}{\partial R}=3 c \frac{\partial\left(R^{2} u\right)}{\partial\left(R^{3}\right)} \text {. }
$$

This term is differenced as

$$
3 \bar{c} \frac{R_{j+2}^{2} u_{j+1}^{n_{1}+1}-R_{j-j}^{2} u_{j-1}^{n+1}}{R_{j+1}^{3} \cdot R_{j-1}^{3}}
$$

in step 2, and in a similar way in step 1. Special treatment of points near the free surface, near the head of the rarefaction wave, and (after collapse) near the center and near the shock front is described in the following sections. 


\section{THE FREE-SURFACE CONDITION}

It is assumed that $\sigma(R, t)$ and $u(R, t)$ are smooth. for $R \geq \xi(t)$. so that the entropy law $(a / \partial t+u d / d R) \tilde{S}$ $=0$ holds near the free surface. Then, for problems in which $\widetilde{S}$ is constant initially, $\widetilde{S}$ is constant throughout the flow until snocks form. (The present discussion needs modification for problems in which $S \neq$ constant initially.) We set

$$
\begin{aligned}
& \sigma(R, 1)=\sum_{p=1}^{\infty} \sigma_{p}(t)\left[R-\left.\xi(t)\right|^{a+p},\right. \\
& u(R, 1)=\dot{\xi}(t)+\sum_{p=c,}^{\infty} u_{p}(t)[R-\xi(t)]^{\beta+p},
\end{aligned}
$$

where a and 8 are positive constants to be determined later. If we substitute $(4.1)$ and $(4.2)$ into $(3.8 b)$ and $(3.8 \mathrm{c})$, with $\mathrm{\partial S} / \partial \mathrm{R}=0$, then, writing only the lowest order terms and replacing the others by dots. we have

$$
\begin{aligned}
& \dot{a}_{0}(R-\xi)^{\circ} \cdots \\
& +u_{0} \sigma_{0}\left(a+\frac{r-1}{2}\right)(R-\xi)^{B+a-1}+\ldots=\ldots \\
& \ddot{\xi}+\ldots+\dot{u}_{0}(R-\xi)^{B}+\ldots+u_{0}^{2} \theta(R-\xi)^{28-1}+\ldots \\
& +\frac{\gamma-1}{2} \sigma_{0}^{2} a(R-\xi)^{2 a-1}+\ldots=0
\end{aligned}
$$

In order to achieve cancellation of the lowest order terms in (4.3). 3 must be $=1$; then, the second and third terms indicated in (4.4) are of higher order, and I 6 achieve canceilation of the first and fourth terms, a must be $=1 / 2$, and we find

$$
\ddot{\xi}+\frac{r-1}{4} \sigma_{0}^{2}=0 .
$$

With or $=1 / 2,(4 . !)$ shows that $\sigma^{2}\left[=\sigma(R, t)^{2}\right]$ is a power series in $R-\xi$, sterting with $\sigma_{0}^{2}(R-\xi)$; hence,

$$
\ddot{E}=-\left.\frac{r-l}{4} \frac{\partial\left(o^{2}\right)}{\partial R}\right|_{R=\xi} .
$$

That is the boundary condition at the free surface; it connects the inward acceleration of the free surface with the rate at which $\sigma^{2} \rightarrow 0$ as the free surface is approached. in the code, $\partial\left(\sigma^{2}\right) / \partial \mathrm{P}$ is approximated by two difference quotients containing values of $\sigma^{2}$ at $\xi$ (where $\sigma^{2}=0$ ) and at nearby net points and is then extrapolated back to $R=\xi$. Enuation (4.5) is implied by Eq. (3.6) of Ref. 7 .

When $\sigma$ has beer calculated at all regular net points at time $t=t^{n+1}$ by the method of Sec. III, Eq. $(4.5)$, for $t=t^{n+1}$, contains two unknowns $\xi^{n+1}$ and $\ddot{\xi}^{n+1}$, tecause $\xi^{n+1}$ enters into the approximation to $\partial\left(\sigma^{2}\right) / \partial R$. That equation is solved, together with the two additional equations

$$
\begin{aligned}
& \xi^{n+1}=\xi^{n}+\Delta i / 2\left(\dot{\xi}^{n}+\dot{\xi}^{n+1}\right) \\
& \dot{\xi}^{n+1}=\dot{\xi}^{n+1}=\dot{\xi}^{n}+\Delta t / 2\left(\ddot{\xi}^{n}+\ddot{\xi}^{n+1}\right)
\end{aligned}
$$

for the unknowns $\xi^{n+1}, \dot{\xi}^{n+1}$, and $\ddot{\xi}^{n+1}$ by Newton's interative procedure. (Only one or two iterations are required at each time step.)

It is easily verified that the boundury condition (4.5) at the free surface is satisfied by the self-similar solutions of the flow equations disclissed in Sec. VII. by virtue of the first equation of the pair (7.9).

The free-surface boundary condition on the variable $u$ has already been incorporated in (4.2); it is

$$
u(\xi, t)=\dot{\xi}(t) .
$$

\section{FITTING THE HEAD OF THE WAVE AND THE FREE SURFACE}

The singularity at $R=\eta(t)$ is of a very mild kind. and the only boundary condition or joining condition needed for the differential equations is the continuity of the function values. Rather little harm is done if this singularity is ignored completely in the calculation: the main effect of so doing is loss of the second-order accuracy of the finite difference equations for that interval that contains the front. at which the second derivatives are infinite. However. it is very easy to treat the singularity correctly. and to do so costs almost nothing cornputationally - it costs less than nothing in the present problem because it obviates the necessity of any computations whatever for $R>\eta(t)$.

A portion of the computational net near the rarefaction head is shown in Fig. 5-1. Whenever the path of the rarefaction head is in either of the positions indicated as (a) and (b) in the figure, that is. lies to the right of a centered point like $x_{2}$ but cuts through the net rectangle that contains that point. values of the flow quantities at $x_{2}$ are obtained by the following special procedure in order that they may then be used in the regular Lax-Wendroff step? to yield the values at the point indicated by the circle on the line $n+1$ : A special Lax-Wendroff step 1 is 


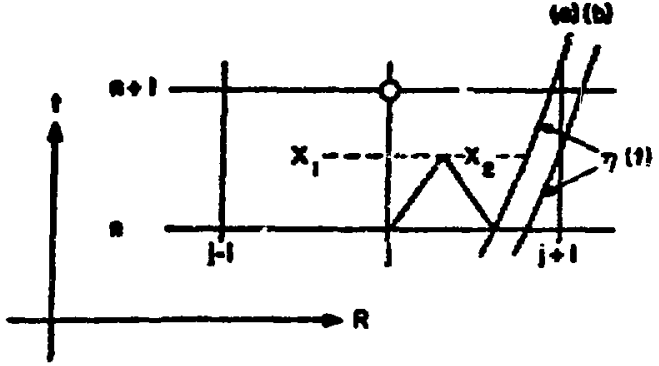

Fig. 5-1.

Treutment of the (mild) singularity at the head of the rarefaction.

performed using the triangle indicated: it is the same as the normai Lax-Wendroff step 1 except that the base of the triangle has been reduced from $I R$ to $\eta\left(t^{n}\right)-R_{i}$. This step gives values of the flow quantities at the tip of the triangle: they are then obtained at $x_{2}$ by quadratic interpolation on $R$ using also the known values at $x_{1}$ and at the rarefaction head itseif.

If the head lies in a position like (b), new values of the flow quantities are needed also at the net pcint $j+1$ that has just been uncovered by the motion of the head; those values are obtained by linear or quadratic interpolation on $R$ at time $t^{n+1}$.

The procedure for determiniing the flow quantities near the free surface is similar, once the motion of the free surface is kn:wn. The sequence is: The moditied Lax-Wendroff step 1 uses the ald position $\xi\left(t^{n}\right)$ of the free surface; the interpolation at $t^{n+1 / 2}$ uses an extrapolated value of $\xi\left(t^{n+1 / 2}\right)$; the LaxWendroff step 2 can then be performed at all ordinary net points, and therefrom the final position $\xi\left(t^{n+1}\right)$ of the free surface is determined as described in the preceding section, followed by interpolation of the flow quantities, at $t^{n+1}$, if needed for an uncovered net point.

\section{SOME NUMERICAL RESULTS FOR THE RAREFACTION PHASE PRIOR TO COLLAPSE}

Several calculations were made, for $\gamma=3.0$, using the methods described in the three preceding sections. Curves of $c$ vs $R$ at four values of $t$ are given. for a calculation in which $\Delta R$ was $=0.02$, in Fig. 6-1. Except where shown, the calculated points lie on the curves drawn within the accuracy of the drawing. The steepening of the gradient at the free surface, which causes the inward acceleration, is cleariy visible.

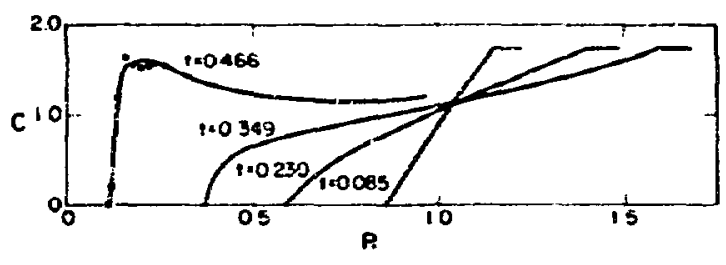

Fig. 6.1.

Prugress of the cavity collapse wace, for $\mathrm{p}=3$.

Except for those shoun. calculated points are within the curces as drawn.

As a provisional measure of overall accuracy, the total energy. kinetic plus internal, of the fluic hetween $R=\xi(t)$ and $R=\eta(t)$ was computed and compared with the initial energy $\left|4 \pi / 3\left(\eta^{3}-\xi^{3}\right) p / \gamma-1\right|$ of that same fluid. The percentage errors are given in Table I (together with the corresponding errors for a calculation with $\perp \mathbb{R}=$ 0.0025 ). A comparison calculation was made with the standard Eulerian equations, with no fitting or boundary condition on the free surface, but with the cavity initially filled, as is often doue. with fluid at .9 very low density and pressure $l=2 \times 10^{-4}$ and $8 \times 10^{-12}$ in units of the initial density and pressure outside the cavity). Comparison of the second and third columns of the table shows that. by this particulis measure of the arcuracy. the errors are reduced by a factor $50-200$ by the methods described in the pieceding sections. Most of the improventent results from the proper treatment of the free surface: the special choice of dependent variables given in Sec. III gives oniv an additional improvement by a factor 2-3.

\section{SOME SELF-SIMILAR SOLUTIONS OF THE BOUNDARY-VALUE PROBLEM}

If the initial conditions at $t=0$ are ignored, one has a boundary-value problem cosisisting of the partial differential equations (3.8) for $R>\xi(t)$ together with the free-surface condition at $R=\xi(t)$. For a $\gamma$ law fluid. for which $\sigma$ and $c$ differ merely by $a$ numerical constant. that problem contains no chararteristic length or time. hence is likely to have self-similar soluticas, i.e., solutions such that the functions of $R$ obtained at any distinct instants $t_{\text {, }}$ and $t_{2}$ become identical if all lengths, velocities, and other cuantities are suitably rescaled between the two instants. (Such solutions can be obtained by solving ordinary differential equations, as shomn below.) In the literature, one often sees arguments claiming to show that the solution of the full initial brundary-value problem, though not self-similar 
TABLE I

ERROR OF TOTAL ENERGY OF RAREFACTION FAR

$r \cdot 3.0$

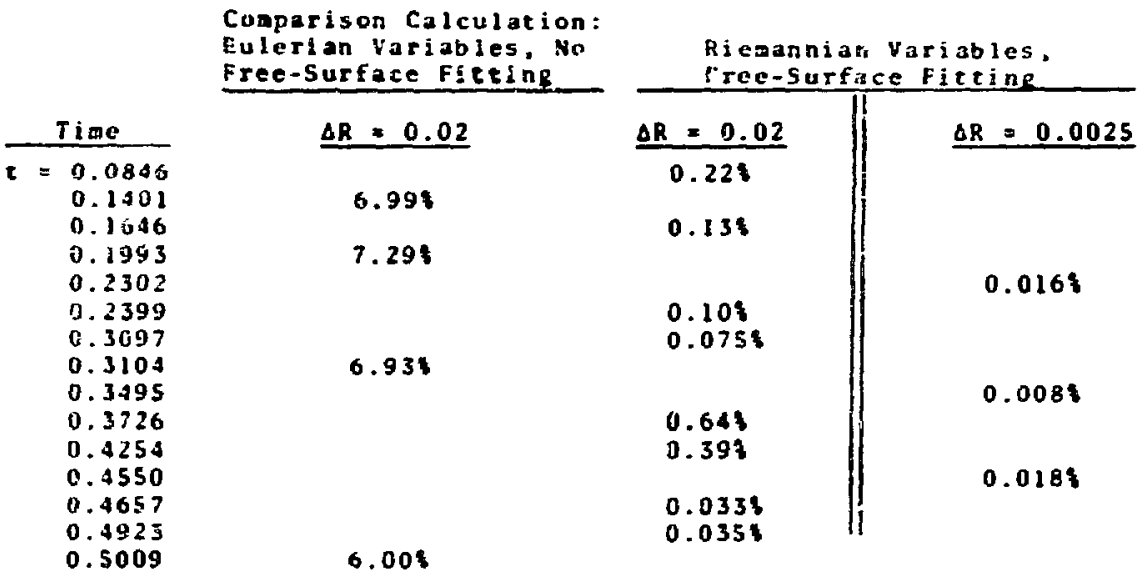

(the initial conditions introduce a characteristic iength $\left.R_{,}\right)$, is asymptotically self-similar near rohlapse, i.e., for $t_{0}-t \ll t_{0}$ and $R \ll R_{w}$. Although we frel there is reason to doubt the general validity of those arguments, we have investigated the self. similar solutions of the boundary-value problem. and we have attempted to examine numerically the solution of the full problem asymptotically near collapse to see whether it has a similarity property. The results so far are inconclusive. but further work is planned. The goal of such work is to be able to patch together numerical calculations just before and just after collapse by solutions obtained from the similarity theory.

For application to the cavity-collapse problem. the rlars of self-similar solutions is further restricted by the assumption of isentropy, which introduces $a$ characteristic constant entropy $S_{0}$. (That is to be contrasted with the assumption made in the Guderley-Butler shock-collapse problem, in which there is a characteristic constant density $\rho_{a}$ - the density of the stationary fluid into which the collapsing shock is running. The two sets of assumptions are cont rasted in Table $\mathrm{Il}$ - they lead to different systems of ordinary differential equations.)

The similarity assumptions are these: First, there are positive constants $A$ and $\delta$ such that the position of the free surface is given by

$$
\xi(t)=A\left(t_{0}-t\right)^{\delta}
$$

In the discussion of the gelf-similar solutions. $t$, could be taken $=0$, but we retain the notation of the preceding sections in the interest of returning later to the full initial boundary-value problem.) Then. a dimensionless variable $\eta$ is deñned:

$$
\eta=\frac{R}{A\left(\tau_{0}-t\right)^{6}}
$$

the region of interest is $n \geq 1 ; \eta=1$ corresponds to the free surface. It is then assumed that the dependent variables depend on $R$ and $t$ only through $\eta$. afier suitable scaling

The dependent varitisles could he chosen as $a$ and $u$, or as $c$ and $u$. since. "or a $\gamma$-law fluid, $\sigma$ and $c$ diffe: merely by a constant. We make the latter choice. in conformity with most of the literature. Since $c$ and $u$ have the dimensions of velocity. they can be writien as

$$
\begin{aligned}
& c(R, T)=R C(\eta) /\left(T_{n}-t\right)=A\left(t_{0}-t\right)^{\delta-1} \eta C(\eta) \\
& u(R, t)=R V(\eta)_{i}\left(t_{0}-t\right)=A\left(t_{0}-t\right)^{\delta-1} \eta V(\eta) .
\end{aligned}
$$

where $\mathrm{C}$ and $\mathrm{V}$ are dimensionless functions, called the reduced sound and material speeds. When the expressions (7.3) are substituted into (3.8). with $\widetilde{\mathrm{S}}=$ const, the explicit dependence on $R$ and $t$ cancels out, and the following system of ordiuary differential equations is obtained: 
TABLE II

SIMILARITY ASSUMPT IONS

$$
\eta=\frac{R}{A\left(t_{0}-t\right)^{6}}
$$

$$
\begin{gathered}
\text { Collapsing Shock } \\
\text { (Guderley, Butler, etc.) } \\
c=A\left(t_{0}-t\right)^{\delta-1} C(n) \\
u=A\left(t_{0}-t\right)^{\delta-1} V(n) \\
P=P(n) \\
{\left[e \cdot g \cdot, P(1)=\frac{Y+1}{Y-1} p_{0}\right]}
\end{gathered}
$$

Consequence:

$$
s=A^{2}\left(t_{0}-t\right)^{2 \delta-2} S(n)
$$

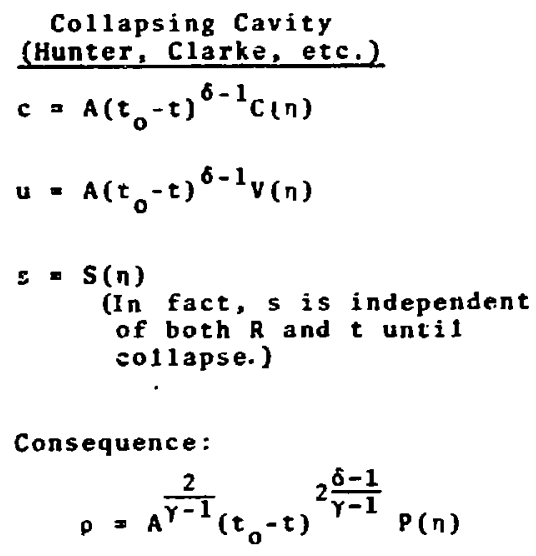

sionally equivalent substitution $c(R, t)=\xi C_{1}(\eta)$, $u(R, t)=\xi v_{1}(\eta)$. The possibiiity of putting the equations into autonomous form is a consequence of the dimensional properties of the fluid-dynamical equations and can be seen as follows: First, we take $A=i$ and $t_{0}=0$ in this paragraph, so that $\eta=$ $R(-t)^{-\delta}$. We write

$$
\frac{C^{\prime}(n)}{V^{\prime}(n)}=\Psi(C(n), V(n), n)
$$

where

$$
د=د(C, V)=(V+\delta)^{2}-C^{2} .
$$

It is noted that the quantity of $d C / d V=C^{\prime} / V^{\prime}$ is a function of $C$ and $V$ and does not depend explicitly on $\eta$. An ordinary differential equation system of the general form (7.4) having that property is called autonomous; it has the advantage for interpretation and presentation of the solutions that a solution curve in the three-dimensional space $\mathrm{C}, \mathrm{V}, \eta$ is uniquely determined by its projection on the $\mathrm{C}, \mathrm{V}$ plane. (Even the explicit appearance of $\eta$ in the left members of (7.4) can be eliminated by taking $\tau=\log$ $\eta$ as the independent variable.)

It should be noted that the autonomous property of (7.4) follows from the particular substitution (7.3) and does not follow, for exampie, from the dimen-
Let $a$ be any positive constant, and let $b=a^{1-\delta}$. Now,

$$
\frac{C^{\prime}\left(b_{n}\right)}{V^{\prime}\left(b_{n}\right)}=\Psi\left(c(b n), V(b n), b_{n}\right) .
$$

On the other hand, for $\widetilde{\mathbf{S}}=$ const, it is seen that if $c(R, t)$ and $u(R, t)$ satisfy $(3.8)$, then the functions

$$
\begin{aligned}
\hat{\mathbf{c}}(\mathrm{R}, \mathrm{t}) & =\mathbf{c}(\mathrm{aR}, \mathrm{at}) \\
\hat{\mathbf{u}}(\mathrm{R}, \mathrm{t}) & =\mathbf{u}(\mathrm{aR}, \mathrm{at})
\end{aligned}
$$

also satisfy (3.8), hence the functions

$$
\hat{c}(n)=c\left(\frac{a R}{(-a t)^{\delta}}\right)=c(b n)
$$




$$
\hat{V}(n)=V\left(\frac{a R}{(-a t)^{\delta}}\right)=V(b \eta)
$$

also satisfy (7.6); that is,

$$
\frac{C^{\prime}\left(b_{n}\right)}{V^{\prime}\left(b_{n}\right)}=\Psi\left(C\left(b_{n}\right), V\left(b_{n}\right), \eta\right) .
$$

Since b is arbitrary (if $\delta \neq 1$ ), comparisos with (7.7) shows that $\Psi$ does not depend explicity on $\eta$. (The argument must he modified for $\delta=1$.)

Tquation ( 7.4$)$ can be written as

$$
\begin{aligned}
& n C^{\prime}(n)=\frac{F(C, V)}{\Delta(C, V)} \\
& n V^{\prime}(n)=\frac{G(C, V)}{\Delta(C, V)}
\end{aligned}
$$

where $F$ and $G$, like $J$, are simply polynomials in $C$ and $V$. The boundary conditions at the free surface $(\eta=1)$ are

$$
\begin{aligned}
& C(1)=O \text { (from vanishing pressure). } \\
& V(1)=-\delta \text { (from } u=\xi \text { at } R=\xi \text { ). }
\end{aligned}
$$

The boundary conditions for $\eta \rightarrow \infty$ are discussed below.

In principle, the system (7.8) can be integrated by Runge-Kutta with (7.9) as starting values. However. there is a difficulty that the point with $C$ and $V$ given by (7.9) is a critical point, i.e., a point where the polynomials $F, G$, and $\Delta$ all vanish. A standard analysis shows that, near the critical point, $V$ has the form of a power series in $\eta-1$, while $C$ has the form $\sqrt{\eta-1}$ times a power series. For that and other reasons, the numerical work was done with functions $q(\eta)$ and $v(\eta)$ given by

$$
\begin{aligned}
& q(n)=\frac{2}{\gamma-1} \frac{n}{\sqrt{n-1}} c(n) \\
& v(n)=n v(n)
\end{aligned}
$$

(then, the system no longer has the autonomous formi. but the results will be described in the C.V plane. The complete set of critical points is described below and in Fig. 7-1.

The boundary conditions for $\eta \rightarrow \infty$ come from the requirement that $c(R, t)$ and $u(R, t)$ have definite limiting values, as $t \rightarrow t_{n}$ for fixed $R$, i.e., as $\eta \rightarrow \infty$.
From the definition (7.2) of $\eta$, it is seen that (7.3) can be rewritten as

$$
\begin{aligned}
c(R, t) & =\left(\frac{R}{\left(t_{0}-t\right)^{\delta}}\right)^{1 / \delta} R^{1-1 / \delta} c(n) \\
& =\left(A_{n}\right)^{1 / \delta} R^{1-1 / \delta} C(n) \\
u(R, t) & =\cdots \\
& =\left(A_{n}\right)^{1 / \sigma_{R}}{ }^{1-1 / \delta} v(n) .
\end{aligned}
$$

Hence, as $\eta \rightarrow \infty$,

$$
\mathrm{C}(\eta), \mathrm{V}(\eta) \sim \eta^{-1 / \delta}
$$

Examination of (7.4) shows that if $\mathrm{C}$ and $\mathrm{V} \rightarrow 0$, as $\eta$ $-\infty$, then they behave asymptotically according to (7.11); hence the boundary condition is that $\mathrm{C}$ and $\mathrm{V}$ $\rightarrow 0$.

For most values of $\gamma$ and $\delta$ in the relevant ranges, the system (7.4) has nine critical points, of which six are shown in Fig. $7-1$ for $\gamma=3, \delta=0.7$ and the remaining three are obtained by reflection in the $\mathrm{V}$ axis. For the general classification of critical points, the reader is referred to Birkhoff and Rota, ${ }^{8} \mathrm{p} .130 \mathrm{ff}$. A little algebra shows that, in the present problem, if either $F$ or $G$ vanishes along with $\Delta$, then the other vanishes ton. That simplfies locating and classifying the critical points.

Factorization of the denominator $\Delta(C, V),(7.5)$, of the expressions (7.8) shows that, along any solution curve in the C.V plane, the direction of change of $\eta$ reverses, as the curve crosses either of the lines $V+\delta$ $= \pm C$, one of which is shown dashed in Fig. 7-1, unless $F$ and $G$ also vanish at the same time. According

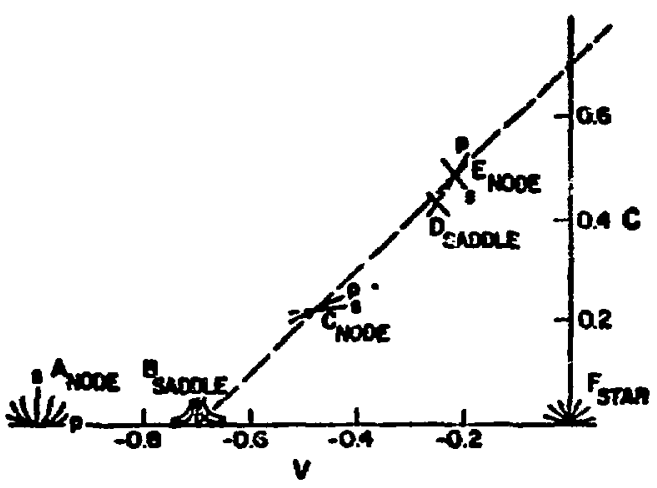

Fig. $7-1$.

Critical puints of $C=C(V)$ in the upper halfplane, for $\gamma=$. 
to the boundary condition given above, the solution curve starts from the saddle point $B$ in an upward direction and terminates, for $\eta \rightarrow \infty$, at the star point $F$; hence, in order that $\mathrm{C}$ and $\mathrm{V}$ be single-valued functions of $\eta$, the curve must cross the dashed line either at the node $\mathrm{E}$ or the node $\mathrm{C}$. Numerical integration with third-order Runge-Kutta for various values of $\delta$ shows that, when a complete solution is obtained at all, it goes through point $\mathrm{C}$, not $\mathrm{E}$.*

The situation is in marked contrast with that for the corresponding collapsing shock problem studied by Guderley and Butler, where the point corresponding to $\mathrm{C}$ is a saddle point, instead of a node. QQualification is necessary, because our studies have been made for $\gamma=3.0$, while those of Guderley and Butler were for $1<\gamma<2$; detailed studies of both problems for general $\gamma$ will be presented elsewhere.) In the case of a node, all solution curves that come reasonably close are drawn into the node, and all, with one exception, pass through it in the direction indicated by the letter " $p$ " (for "primary") in Fig. 71 ; the one exception takes the direction " $s$ " (for "secondary"); whereas, in the case of a saddle point, only the curve that is aimed in precisely the right way passes through the point at all; all other solutions deviate either to the right or to the left. Consequently, in order to get a solution at all, for the collapsing shock problem, the value of $\delta$ has to be precisely chosen, whereas there is range of admissible $\delta$ 's for the collapsing cavity problem. In either case, once the dashed line has been crossed, the solution is drawn automatically to the star point $F$ at the origin, and the terminal boundary condition is satisfied.

The results described from now on are all for $\gamma=3.0$.

For $\delta$ in the interval $0.666667<\delta<0.708542$, the solution curve arrives at the node $\mathrm{C}$ along the primary direction, but there are many curves leaving $\mathrm{C}$ to the right: one starting in the secondary direction, as seen most clearly in Fig. 7-2, for $\delta=0.68$, and $a$ one-parameter family of curves starting in the primary direction, as illustrated by the upper three curves of Fig. 7-3, for $\delta=0.70$. (The arrows indicate the direction in which the curves were calculated; in particular, the lowest one was calculated both

*At least for $\gamma=3$. For the collapsing shock problem (to be published), the situation reverses at a critical value of $\gamma$ (near 1.9, but different for cylindrical and spherical geometry). It remains to be investigated whether the same is true for the collapsing cavity proulem, and, if so, whether the critical values of $\gamma$ are the same.

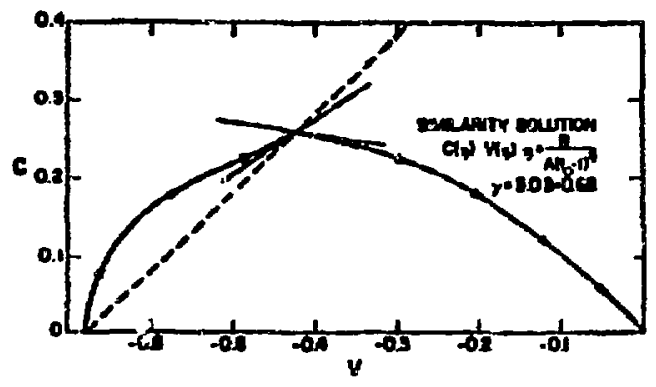

Fig. 7-2.

$A$ similarity solution for $\delta=0.68, \gamma=3$.

forward and backward, by reversing the sign of $\Delta \eta$ in the Runge-Kutta method; the direction of increase of $i$ is from left to right along all the curves.) The curves of the one-parameter family (for given $\delta$ ) pass through $\mathrm{C}$ with change of direction, but generally without discontinuity of higher derivatives.

In the special case $\delta=0.708542$, the curve comes to $\mathrm{C}$ along the secondary direction and, if continued so as to leave in the same direction, passes through $\mathrm{C}$ with continuity of all derivatives and in fact analytically. For certain special values $\delta$ in $(0.666667,0.708542)$, one curve of the one-parameter family referred to is also analytic at $\mathrm{C}$. That appears to be a rather intricate Diophantine affair and is discussed in detail by Hunter. ${ }^{6}$ who, however, rejects those solutions for reasons that are rather hard to follow.

An argument given by Hunter claims to show that only those solutions analytic at $\mathrm{C}$ can appear asymptotically in a physical problem. That argument seems doubtful to us for the following reason: In the first place, any of the curves in the $\mathrm{C}, \mathrm{V}$ plane discussed above, including those of the two-parameter family (where $\delta$ is now regarded as the second parameter) lead to an acceptable solution of the partial differential equations of fluid dynamics, by

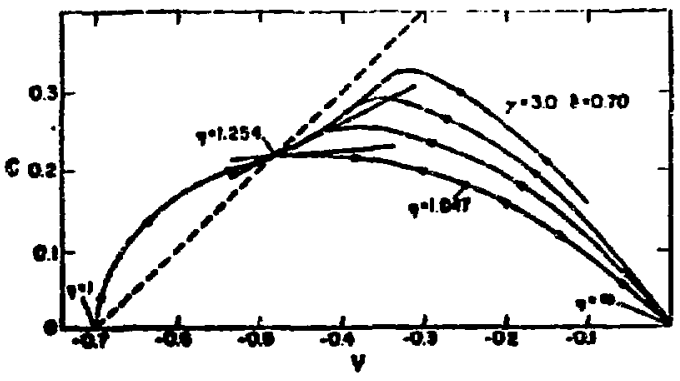

Fig. 7-3.

Some of the similarity solutions for $\delta=0.70, \gamma$ $=3$. 
means of Eqs. (7.2) and (7.3). Since the fluiddynamical equations are hyberbolic, there is no requirement of analyticity; in fact, jumps of the various derivatives of the flow quantities can be propagated along the characteristics; the first derivatives are discontinuous at the head of a rarefaction wave, and the functions themselves can be discontinuous for a weak solution. Now, the solution of the full initial boundary-value problem of the cavity-collapse problem is indeed analytic; it can be shown (again by consideration of the characteristics), but it does not follow that the selfsimilar solution to which it converges must be analytic.

If $\eta_{0}$ is the value of $\eta$ at the node $C$, then the curve $\eta=\eta_{0}$ in the $R, t$ plane, namely the curve

$$
\mathrm{R}=\mathrm{A} \eta_{0}\left(\mathrm{~T}_{0}-\mathrm{t}\right)^{\delta} \text {, }
$$

is characteristic; it is the path of an incoming spherical sound wave that just catches up with the free surface at collapse. For determining how the free surface itself collapses, nothing outside that sound wave has any influence, hence the part of the solution curve to the right of the dashed line in the figures has no effect, un: il after collapse.

The properties of the critical point $\mathrm{C}$ as a function of $\delta$ in $(0,1)$ (still for $\gamma=3.0$ ) can now be summarized. (It always lies on the dashed line in the figures.) For $0.708542<0.711405$, the solution curve comes to $\mathrm{C}$ alung the primary direction from above and to the right. and crosses the dashed line first, hence is unacceptable. For $\delta>0.711405, \mathrm{C}$ is a spiral point, hence again the solution curve crosses the dashed line (in fact infinitely often) before arriving at $C$. For $\delta<0.666667$, the point $C$ disappears (it merges with $F$, then becomes complex, as $\delta$ decreases). Hence, we are left with the interval (0.666667, 0.708542).

\section{THE SIMILARITY SOLUTION AFTER COLLAPSE; THE OUTGOING SHOCK}

At the instant of collapse $\left(t=t_{0}\right)$, according to (7.8) and the equations just preceding it and the relations $p=s \rho \gamma, c^{2}=\gamma p / \rho$, the flow quantities vary as inverse powers of $R$, namely,

$$
\begin{aligned}
& c\left(R, t_{0}\right)=c_{00} R^{-\frac{1-\delta}{\delta}} \\
& u\left(R, t_{0}\right)=u_{o 0} R^{-\frac{1-\delta}{\delta}}
\end{aligned}
$$

$$
\begin{aligned}
& p\left(R, t_{0}\right)=o_{0} R^{-\frac{2(1-\delta)}{\delta(\gamma-1)}} \\
& p\left(R, t_{0}\right)=p_{00} R^{-\frac{2 Y(1-\delta)}{\delta(\gamma-1)}} .
\end{aligned}
$$

The infinite pressure gradient at $\mathbf{R}=\mathbf{0}$ starts an outgoing shock, and wo look for a self-similar solution for that shock and the flow behind it. The problem was considered by Hunter, ${ }^{6}$ but only in the isentropic approximation.

As in Sec. VII, the flow quantities are written as functions of a similarity variable, each multiplied by a power of $t-t_{0}$. As similarity variable, we choose

$$
\hat{n}=\frac{R}{A\left(t-t_{0} j^{\delta}\right.}=e^{-i \pi \delta} n .
$$

(This choice makes $\hat{\eta}$ reai, for $t \geq t_{0}$. Since only $\log \eta$ appears in the ordinary differential equations, the extra factor $\mathrm{e}^{-i \pi \delta}$ is irrelevant.) The flow ahead of the shock, for $t \geq t_{0}$, is simply the smooth continuation of the flow found in Sec. VII; hence, the value of $\delta$ has to be the same as in that section, to give the same behavior (8.1) for $t=t_{b}$. It then follows that the power of $t-t_{0}$ appearing in each flow quantity must be the same as in Sec. VII, because the compression ratio $\rho_{2} / \rho_{1}$ across the shock, the corresponding pressure ratio $\mathrm{p}_{2} / \mathrm{p}_{1}$, velocity ratio $u_{2} / u_{1}$, and "entropy" ratio $s_{2} / s_{1}$ (where $s=p p^{-\gamma}$ ), and so on, are all independent of $t$ for a self-similar solution. Therefore, we write [compare with (7.3)]:

$$
\begin{aligned}
& c(R, t)=\frac{R}{t_{0}-t} C(\hat{n})=-A\left(t-t_{0}\right)^{\delta-1} \hat{n} C(\hat{n}), \\
& u(R, t)=\frac{R}{t_{0}-\tau} V(\hat{n})=-A\left(t-t_{0}\right)^{\delta-1} \hat{n} V(\hat{n}), \\
& S(R, t)=S(\hat{n}),
\end{aligned}
$$

for $t>t_{0}$. The functions $C, V$, and $S$ can have jumps at the point $\hat{\eta}=\hat{\eta}^{*}$ where the shock occurs. (Note that $c$ and $C$ have opposite signs, as do $u$ and $V$; that is unfortunate, but it obviated recoding some of the computer programs.)

In the similarity variables, the entropy equation

$$
\frac{\partial S}{\partial t}+u \frac{\partial S}{\partial R}=0
$$


takes the form

$$
\left[\delta+V(\hat{\eta}) \mid S^{\prime}(\hat{\eta})=0 .\right.
$$

It can be shown that $V(\hat{\eta})$ cannot be $\equiv-\delta$. In fact, $V(\hat{\eta})$ cannot be $=-\delta$ immediately behind the shock, at $\hat{\eta}=\hat{\eta}^{*}$, no matter what value $\hat{\eta}^{*}$ has; that follows from the numerical values of the self-similar solution already obtained for the flow ahead and the Rankine-Hugoniot shock conditions. Therefore, $S^{\prime}(\hat{\eta}$ $=0$, and $s(R, t)$ has a constant value $s_{2}$ in the flow behind the shock, which is, of course, not necesarily equal to the constant value $s_{1}$ ( $=1$ in Sec. VII) ahead of the shock; in fact, $s_{2}>s_{1}$ because a shock always increases the entropy.

Since the entropy is constant, the ordinary differential equations (7.4) and (7.8) hold also behind the shock. In Fig. 10-4, two solution curves are shown in the $\mathrm{C}, \mathrm{V}$ plane: the curve $\mathrm{C}$, consisting of the parts $C_{1}, C_{2}$, and $C_{3}$, which describe the flow ahead of the shock, and the curve $C^{\prime}$, which describes the flow behind it. The shock is a jump from a point $P_{o}$ on the first curve to a point $P_{0}^{\prime}$ on the second.

The boundary conditions at $\hat{\eta}=0$ for the curve $C^{\prime}$ come from conditions at the center of the system. For $R=0$, the velocity $u$ vanishes by symmetry, while the sound speed $c$ assumes a positive value. Hence, by (7.3), $V(0)$ is finite, while $\mathbf{C}(\hat{\eta}) \rightarrow \infty$, as $\hat{\eta}$ $\rightarrow 0$. By letting $\mathrm{C}(\hat{\eta}) \rightarrow \infty$ in the second differential equation (7.4), we find that

$$
V(0)=-2(1-\delta) / 3(\gamma-1)
$$

That suffices to start the curve $C^{\prime}$ at very small $\dot{\eta}$, hence very large negative $C(\hat{\eta})$.

Let $\hat{y}$ be so normalized on $C^{\prime}$ (it can be multiplied by an arbitrary constant), that it has the value $\dot{\eta}^{*}$ at $P^{\prime}$. Then, the constant $A$ in (8.2) has the same value on both curves, because the coordinate $R$ of the shock is the same when viewed from in front of the shock or behind it; the shock's position is

$$
R_{k h}=A \hat{\eta}^{*}\left(t-t_{n}\right)^{\delta},
$$

and its speed is

$$
\dot{\mathrm{R}}_{\mathrm{sh}}=\delta A \hat{\eta}^{*}\left(\mathrm{t}-\mathrm{i}_{\mathrm{a}}\right)^{\delta-1} .
$$

For the Rankine-Hugoniot jump conditions across the shock, let subscripts 1 and 2 denote values just before and just behind the shock, respectively. The conditions are

$$
\begin{aligned}
& \left(\dot{\mathrm{R}}_{\mathrm{sh}}-\mathrm{u}_{1}\right) \mathrm{p}_{1}=\left(\dot{\mathrm{R}}_{\mathrm{sh}}-\mathrm{u}_{2}\right) \mathrm{p}_{2}=\sqrt{\frac{\mathrm{p}_{2}-\mathrm{p}_{1}}{\frac{1}{\rho_{1}}-\frac{1}{\rho_{2}}}}, \\
& \frac{\mathrm{p}_{2}}{\mathrm{p}_{1}}=\frac{\theta x-1}{\theta-x}, \text { where } x=\frac{p_{2}}{p_{1}}, \theta=\frac{r+1}{r-1} .
\end{aligned}
$$

In the similarity variables, these equations take the form

$$
\begin{aligned}
& x=\frac{r \phi_{0}}{\delta \phi_{0}+\theta+1} \text {, where } \phi_{0}=\frac{\left(v_{1}+\delta\right)^{2}}{c_{1}^{2}} \\
& c_{2}=c_{1} \sqrt{\frac{\theta x-1}{(\theta-x) x}} \\
& v_{2}=\frac{v_{1}+\delta}{x}-\delta .
\end{aligned}
$$

The numerical procedure for locating the jump is this: For each point $P$ on $C_{3}$ (where $C_{1}$ and $V_{1}$ are known), $C_{2}$ and $V_{2}$ are determined from these equations as target values to be attained by the jump, if the jump were to occur at point $P$. Then, for each $P^{\prime}$ on $C^{\prime}$, the value $V_{2}$ of $V$ at $P^{\prime}$ fixes the point $P$ on $C_{3}$ from which the jump would have to start; $P^{\prime}$ is moved along $C^{\prime}$ (upward in the figure) and $P$ correspondingly along $C_{3}$, until condition $(8.9)$ is also satisfied; $\hat{\eta}^{*}$ is then the value of $\dot{\eta}$ at the point $P$ on $C_{3}$, and the shock is completely determined.

Each of the solutions, for $t<t_{o}$, of the twopaiameter family discussed in Sec. VII can be completed for $t>t_{0}$, in the way described here, by a selfsimilar solution containing an emerging shock. An example is shown in Fig. 10-4.

\section{THE FLUID-DYNAMICAL EQUATIONS IN SIMILARITY VARIABLES}

To follow the motion of the free surface and the fluid just behind it near collapse, a special computational net and a special set of difference equations are used for the region $t \approx t_{n}, R \approx 0$. The independent variables are $\eta$ and $t$, instead of $R$ and $t$. where

$$
\eta=R / \xi(t) .
$$


$\xi(t)$ being the position of the free surface, as determined by the calculation itself. Relative to the $\mathbf{R}$ grid, the $n$ grid represents a moving and shrinking frame of reference. The two calculations are coupled by interpolation at intermediate radii, and the whole is called similarity-fitting. The dependent variables are $Q(\eta, t)$ and $V(\eta, t)$ and are related to the Reimann variable $\sigma$ and the fluid speed $u$ by the equations

$$
\begin{aligned}
& \sigma(R, t)=\dot{\xi}(t) \sqrt{\eta-1} \mathbf{Q}(\eta, t) \\
& u(R, t)=\dot{\xi}(t) V(\eta, t) .
\end{aligned}
$$

The factors $\dot{\xi}(\mathrm{t})$ are included to make $Q$ and $V$ dimensionless. (Note 1: It would have heen more reasonable to include a minus sign in those definitions, because $\dot{\xi}<0$, but that was not done. Note 2: The entropy equation was also carried along in the calculation, but vacuously, because only the isentropic case was computed.)

The partial differential equations result from substituting (3.2) into (3.8) and dropping the terms in $\widetilde{\mathbf{S}}$; they are:

$$
\begin{aligned}
& \dot{Q}+\frac{\ddot{\xi}}{\dot{\xi}} Q+\frac{\dot{\xi}}{\xi}[(V-n) \\
& \left.\cdot\left(Q^{\prime}+\frac{Q}{2(n-1)}\right)+\frac{\gamma-1}{2} Q \frac{1}{n^{2}}\left(n^{2} V\right) \cdot\right]=0, \\
& \dot{v}+\frac{\ddot{\xi}}{\dot{\xi}} V+\frac{\dot{\xi}}{\xi}\left[(V-n) V^{\prime}\right. \\
& \left.+\frac{Y-1}{2} Q\left\{(n-1) Q^{\prime}+\frac{1}{2} Q\right\}\right]=0 .
\end{aligned}
$$

where the dot rlenotes $\partial / \partial t$ and the prime $\partial / \partial \eta$. The system is of the form (3.7) and was differenced by the Lax-Wendroff two-step procedure (3.9).

If the how is asymptotically self-similar, then $Q(\eta, t)$ and $V(\eta, t)$ should hecome independent of $t$, as $1 \cdot \operatorname{tr}$.

The free-surface boundary conditions come from (1.5) and (4.7); they are

$$
\left.\begin{array}{l}
\frac{\ddot{\xi}}{\xi}=-\frac{y-1}{4} \frac{\dot{\xi}}{\zeta} Q^{2} \\
v=1
\end{array}\right\} \text { at } n=1 \text {. }
$$

Note: The symbol $V$ is used differently here and in Ser. VIl. To get the quantities $C$ and $V$ of that sec. tion. the present quantities $\sqrt{\eta-1} Q$ and $V$ must be multiplied by $\delta / \eta$.
A consequence of the transformation (9.2) of the dependeni variables is that, whereas $\sigma$ and $u$ were both known in advance at the free surface for the $R$ grid (in particular, $\sigma=0$ ), $Q$ is now unknown there, and an additional equation is needed; it is obtained by setting $\eta=1, \mathrm{~V}=1$ in the firs: equarion of (9.3) and evaluating $(V-\eta) /(\eta-1)$ by L'Hópital's rule Iseting $\eta=1 . V=1$ in the second equation of (9.3) merely gives the first of (9.4) again). We find

$$
\dot{Q}+\frac{\ddot{\xi}}{\xi} Q+\frac{\dot{\xi}}{\xi} \frac{\rho}{2}[\mathrm{r}(V+2)-3\}=0 .
$$

This equation is used (in effect as a special step 2 of the Lax-Wendroff) to advance $Q$ in time at $\eta=1$. The derivative $V^{\prime}$ is obtained at $t^{n+1 / 2}$ from the results of step 1 at $\eta-1=\Delta \eta / 2$ and $3 \Delta \eta / 2$, tcgether with the value $V=1$ at $\eta=1$, by differencing the extrapolation. Since $\mathbf{Q}^{\prime}$ has disappeared, the above equation is not coupled to the equations for advancing $Q$ at the regular net points $\eta-1=k \Delta \eta\{k=$ 1,2...). A more careful treatment provides such coupling. Instead of merely evalusting the differential equation at $\eta=1$, we average it over the interval $(1.1+\Delta \eta)$ with a weight that decreases linearly from 1 to 0 across the interval. (The corresponding effect is achieved in the normal Lax-Wendroff by the averaging that takes place in step 1.) Then, the above equation has an extra term and takes the following form:

$$
\dot{Q}+\frac{\ddot{\xi}}{\dot{\xi}} Q+\frac{\dot{\xi}}{\xi}\left\{\frac{Q}{2}\left(r\left(v^{\prime}+2\right)-3\right)+\frac{1}{3}\left(v_{1}-n_{1}\right) Q^{\prime}\right\}=0 \text {, }
$$

where $\eta_{1}=1+\Delta \eta$ and $V_{1}=V\left(\eta_{1}\right)$. Since the last term it: of smaller order than the others, it is adequate to evaluate $Q^{\prime}$ to first order from the values at $t^{n}$.

The application of (9.4) and (9.5) is similar to the fit ting procedure at the free surface described for the $R$ net in Secs. IV and $V$, but with the following differences: (a) there is no need for a special LaxWendroff step 1 , because the free surface is always at the net point $k=0$; (b) after step $l$ at $k=1 / 2,3 / 2 \ldots$ and the approximation to $\bar{\xi}, \dot{\xi}$. and $\xi$ at $t^{n+1 / 2}$ that result therefrom, and after the regular step 2 at $k=$ $1.2, \ldots . .5 q$. (9.5) is then used to advance $Q$ at $\eta=1$ as described above, and the values of $\dot{\xi}$. and $\xi$ at $t^{n+1}$ are obtained from (9.4): (c) there is no need for interpolations at $t^{n+1}$, because no points of the $\eta$ net are uncovered by the motion of the free surface.

When the method of this section is combined with that of Sec. III. involving a standard $R$ - $t$ net, and the two calculations are coupied by mutua! inter. polations at intermediate radii, the effect is to 
provide a refinement of the $R-t$ net near $t=t_{o}, R=$ 0 . the degree of refinement increasing without limit as collapse is approached. The overall procedure is called zooming, to borrow a term from photograpiny.

The resulting algorithm wes tested by a calculation in which the initial values of $Q$ and $V$ were taken, for an interval $i=\eta_{0} \leq \eta \leq \eta_{k}(=2.0$ or 5.0), from the similarity theory of Sec. VII, for $\delta=$ 0.708542 . The computed $Q$ and $V$ were very nearly independent of $t$ over a very large number of cycles, as they ought to be, while $\xi(t)$ and $\xi(t)$ varied as shown in the log-log plot of Fig. 9-1. It is seen that the values iie on a straight line with slope $-(1-\delta) / \delta$, as they should.

For $\eta>\eta c$ ( $\eta c$ is the value of $\eta$ at the critical point through which the solution of the ordinary differential equation passes; $\eta_{c}=1.22195$ for $\delta=0.708542$ ), both characteristics of the system (9.3) slope to the right; they both represent signals moving away from the region near the free surface. One consequence is that in the test calculation just referred to, one must not impose a boundary condition at $\eta=\eta_{k}$; the boundary value must float freely with the solution of the differential equation. To achieve that, we calculated the boundary values by one step of the socalled Courant-Isaacson-Rees method, which consists of writing the equation in characteristic form. following each characteristic back from $\eta=\eta \mathrm{h} . \mathrm{t}=$ $t^{n+1}$ to a point between $\eta_{k-1}$ and $\eta_{k}$ at $t=t^{n}$, obtaining a value of the Riemann invariant at that point by linear interpolation on $\eta$, and then using that value of the invariant at $\eta_{k}, t^{n+1}$.

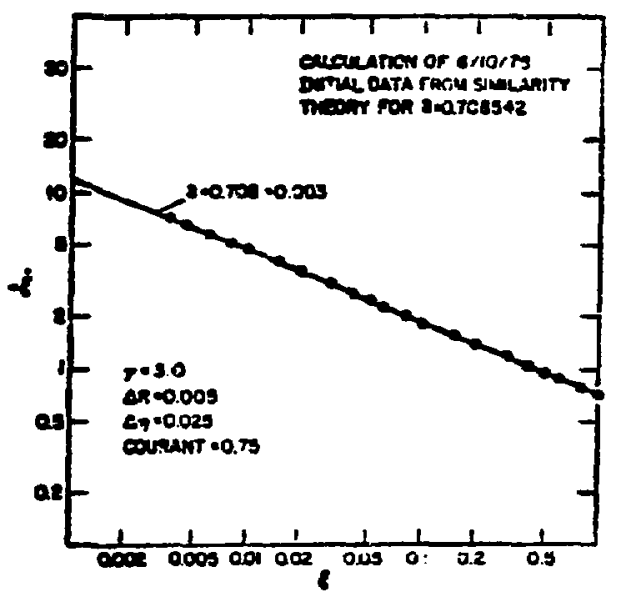

Fig. 9-1.

Agreement of the solution of the partial differential equations with the similarity solution, when the initiul data are taken from the similarity solution.

\section{$X$. THE ASYMPTOTIC BEHAVIOR OF THE CAVITY-COLLAPSE PROBLEM}

In view of the large number (in fact, twoparameter femily) of self-similar solutions found in Sers. VII and VIIl. the question arises, to which of them the solution of the full cavity-collapse problem is asymptotic. and indeed whether the solution of that problem is asymptotically self-similar at all. The same questions arise about other problems of cavity collapse, such as ones in which the fluid is in. itially moving inward, ones with spherical layers of different material, and so on. To begin investigating those questions, a few calculations have been made with the zooming method described in the preceding section, for the present problem as described in Sec. II. One such calculation, for $\gamma=3.0$, which used 400 points in the $R$ net and 700 in the $\eta$ net. will now be briefly analyzed, and will be referred to simply as the full calculation.

One method, in principle, for testing the asymptotic self-similarity of the solution obtained by the full calculation is to plot $\dot{\xi} \log -\log$ vs $\xi$; the graph should be asymptotically a straight line with slope $-(1-\delta) / \delta$. If the solution were truly self-similar, that would be as good as any other method of test. In practice, it is unsatisfactory, because, as stated in Secs. VII and IX. the motion of that part of the fluid corresponding to the interval $\left\{1, \eta_{c}\right)$ of the variable $\eta_{\text {, }}$ a part having vanishing mass in the limit $t=t_{o_{n}}$ is unaffected hy the motion of all the fluid outside, until after collapse; hence, its motion is not necessarily representative of that of the bulk of the fluid. The $\log -\log$ plot of $\dot{\xi}$ vs $\xi$ is given in Fig. 10-1, and it is

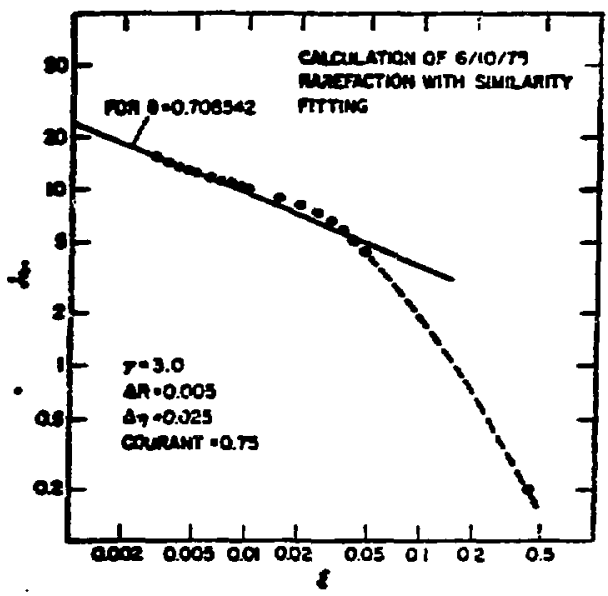

Fig. 10-1.

Weak approach of $\xi$ us $\xi$, from solution of the partial $\because$ ferential equations, to the similarity solution. 
seen that the self-similar property is established, at best, only late in the flow. A much better method is the following.

If the solution is assumed to be asymptstically self-similar, then, for $t$ sufficiently near $t_{o}$ and for an interval $\left(R_{1}, R_{2}\right)$ of the radial coordinate $R$ such that

$$
\xi(t) \ll R_{1}, R_{2} \ll \eta(t),
$$

the flow variables ought to be approximately equal to certain inverse powers of $R$, as given by (8.1). To test that assumption, $-u$ is plotted logarithmically against $R$ for two values of $t$ in Figs. 10-2 and 10-3. The values were obtained from the full calculation; some of the points are from the $R$ net and some from the $\eta$ net. The values of $\xi(t)$ are 0.00960 and 0.00299 for the two cases, and the rarefaction head is at $\eta(t)$ $\approx 2.0$. It is seen that, at intermediate radii, the points lie approximately on a straight line, whose slope $($ An be estimated graphically to slightly better than $1 \%$. In this way, empirical values 0.684 and 0.686 of $\delta$ were ubtained for the two cases.

Clearly, a much more detailed study will be required to establish whether the solution is asymptotically self-similar.

Even if the value $\delta=0.685$ is accepted, a choice must still be made among the one-parameter family of self-similar solutions indicated schematically (for $\delta=0.70$ ) in Fig. 7-3, before the outgoing shock can be started by the similarity method of Sec. VIII. It is evident from Fig. 7-3 that the choice must be based on the ratio $\mathrm{C} / \mathrm{N}$ for large $\eta$, i.e., for $\mathrm{R}$ in the interval $\left(R_{1}, R_{2}\right)$ given by (10.1). The full calculation gives $\mathrm{C} / \mathrm{N} \approx 1.0$, which corresponds to the lowest curve of Fig. 7-3, the one that emerges from the nodal point along the secondary direction. With that choice, for $\delta$ $=0.685$, the complete similarity solution for the flow both before and after collapse is given in Fig. 10-4, obtained by the methods of Secs. VII and VIII.

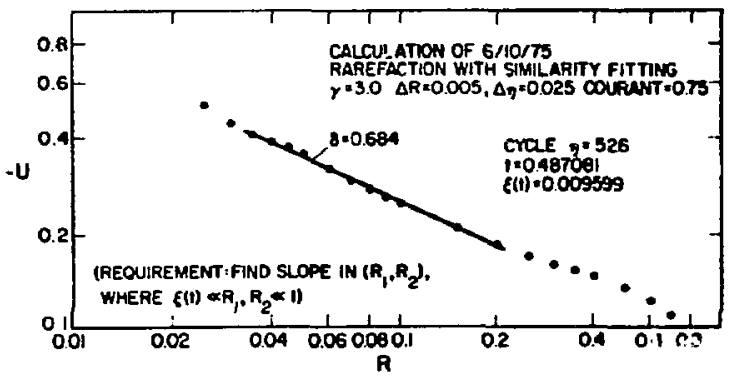

Fig. 10-2.

Approach of $u(R)$ from solution of the partial differential equations to the similarity solution, using the discontinuous initial data, when the free surface is at 0.01 .

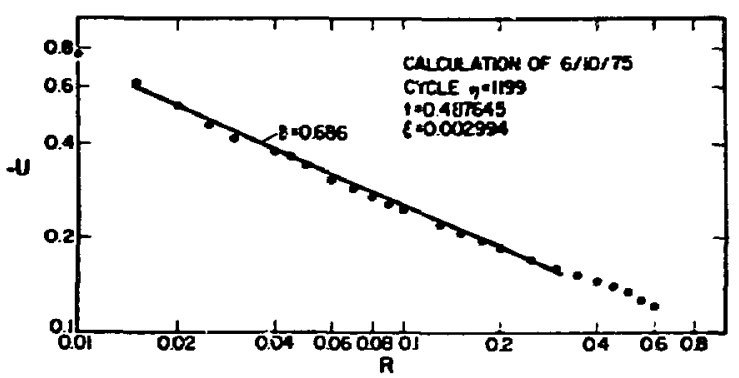

Fig. 10-3.

With the free surface at 0.003 .

In that way, it is tentatively concluded that the outgoing shock travels with a speed $\approx 1.484$ times the speed of the incoming free surface, at a given radius, has Mach number $\approx 1.783$, compression ratio $\rho_{2} / \rho_{1} \approx 1.518$, and "entropy" ratio $\mathrm{s}_{2} / \mathrm{s}_{1}, \approx 1.212$. It can in principle then be followed at later times by the shock-fitting methods of Ref. 3, although that has not yet been done for the present problem.

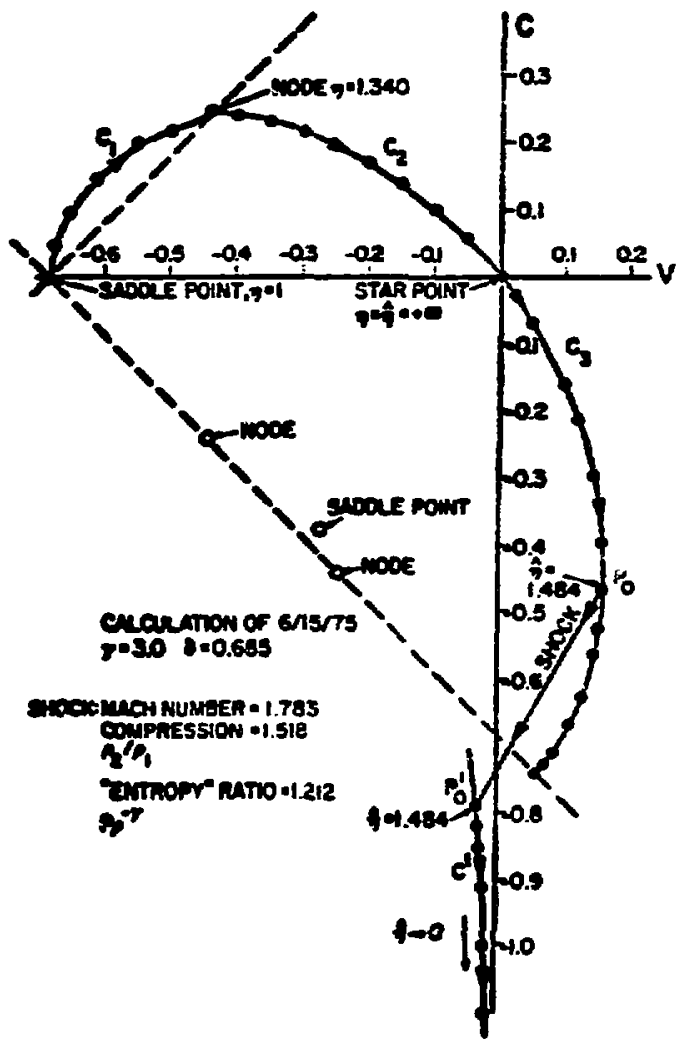

Fig. 10-4.

V.C plane display of an entire similarity solution, for $\delta=0.685, \gamma=3$. 


\section{CONCLUSIONS}

Satisfactory numerical methods have been developed for handling singularities of the first three kinds listed in Sec. II, namely the free surface, the rarefaction head, and the region in between at early times. The methods are described in Secs. III-V and were shown to give about two orders of magnitude greater accuracy than a comparison calculation by conventional methods. The methods that have been developed for singularities of the fourth and fifth kind, namely the cavity collapse at late times and the emergent shock at early times, are described iSess. VII-X; they are somewhat less satisfactory from a theoretical point of view, owing to unresolved questions whether the solution is asymptotically self-similar, and, if so, what the values of the appropriate parameters are.

A method of testing the asymptotic self-similarity of the solution of the full problem is given, which is superior to the perhaps more obvious method of a $\log$ - $\log$ plot of $\xi$ vs $\xi$.

The similarity theory has been developed in some detail. The present work goes beyond that of Hunter ${ }^{6}$ in that the entropy increase at the shock is taken into account. It is shown that there is a twoparameter family of self-similar solutions that contrasts with the collapsing shock problem of Guderley ${ }^{9}$ and Butler, ${ }^{10}$ in which there is only one.

\section{REFERENCES}

1. J. von Neumann and R.D. Richtmyer, "A Method for tiıe Numerical Calculation of Hydrodynamical Shocks," J. Appl. Phys. 29, 232 (1950).
2. R.D. Richtmyer, "Methods for (Genezally Unsteady) Flows with Shock: A Brief Survey," Proc. Third Intern. Conf. on Numerical Methods for Fluid Dynamics, Berlin, July 5-7, 1972 (Springer, Berlin. 1973) Vol. I, pp. 72 ff.

3. R.B. Lazarus and R.D. Richtmyer, "Progress Report on the Shock-Fitting Project," Los Alamos Scientific Laboratory, unpublished data (1975).

4. R.D. Richtmyer and K.W. Mortor, Difference Methods for Initial-Value Problems, Second Ed. (Wiley-Interscience, New York, 1967), pp. 73 ff, 300 ff.

5. R. Courant and K.O. Friedrichs, Supersonic Flow ana' Shock Waves (Interscience Publishers, New York, 1948), pp. $101 \mathrm{ff}$.

6. C. Hunter, "On the Collapse of an Empty Cavity in Water," J. Fluid Mech. 8, 241-163 (1960).

7. H.P. Greenspan and D. Butler, "On the Expansion of a Gas into Vacuum," J. Fluid Mech. 13, 101 119 (1962).

8. G. Birkhoff and G.C. Rota, Ordinary Differential Equations (Ginn and Co., Boston,1961), pp. $130 \mathrm{ff}$.

9. G. Guderley, "Starke kugelige und zylindrische Verdichtungsstöse in der Nahe des Kugelmittelpunktes baw der Zylinderachse," Luftfahrt Forsch.19, 302-312 (1942).

10. D.S. Butler, "Convering Spherical and Cylindrical Shocks," Armament Research Establishment report 54/54 (1954). 